\title{
Contamination Rate of Burnt Necrotic Tissue after Electrocoagulation in Total Knee Arthroplasty
}

\author{
Piti Rattanaprichavej, MD, Artit Laoruengthana, MD, Monton Galassi, MD, \\ Santi Weerakul, MD, Supachok Rasamimongkol, MD \\ Department of Orthopaedics, Faculty of Medicine, Naresuan University, Phitsanulok, Thailand
}

\begin{abstract}
Background: Periprosthetic joint infection (PJI) is one of the commonly found catastrophic complications after total knee arthroplasty (TKA). Preoperative antibiotic prophylaxis, proper skin cleansing, shortened operative time, and sterility of surgical field and equipment are essential to minimize the risk of PJI. Although bacterial contamination of electrocautery tips has been reported, contamination of residual product of electrocoagulation, burnt necrotic tissue (BNT), is not well known. Therefore, we aimed to assess the contamination rate of BNT and association between contaminated BNT and PJI, and risk factors.

Methods: BNTs from 183 patients who had undergone unilateral primary TKA at our institution were retrospectively analyzed. In each patient, three to five specimens of BNT were routinely collected in the operative field of primary TKA. Collecting time was defined as the duration from start of using the electrocautery device to the first collection of BNT.

Results: Culture was positive in eight of 183 patients (4.4\%; contaminated BNT group), and the most commonly isolated organism was coagulase-negative Staphylococcus (62.5\%). The average operative time was $103.1 \pm 44.2$ minutes in the contaminated BNT group and $79.0 \pm 16.7$ minutes in the non-contaminated BNT group ( $p=0.17$ ), and collecting time was $48.0 \pm 44.3$ minutes and 29.7 \pm 17.0 minutes $(p=0.28)$, respectively. None of the patients with contaminated BNT developed PJI, whereas four patients with culture-negative BNT developed PJI within 2 postoperative years.

Conclusions: BNT in surgical field can become a reservoir of contaminating bacteria. However, contamination of BNT was not associated with PJI. Therefore, routine removal of all BNTs may be unnecessary.

Keywords: Knee, Prosthesis-related infections, Arthroplasty, Electrocoagulation, Surgical instruments
\end{abstract}

Total knee arthroplasty (TKA) is one of the most successful and effective surgical procedures in modern orthopedic history. It can relieve pain and symptoms, restore joint function, increase range of motion, and improve quality of life among patients with end-stage knee arthropathy. ${ }^{1,2)}$ However, periprosthetic joint infection (PJI) is a common catastrophic complication of TKA. ${ }^{3)}$ It is consequently one of the common causes of revision arthroplasty ${ }^{4}$ and the leading cause of morbidity after TKA, with the mortality

Received April 10, 2019; Accepted July 3, 2019

Correspondence to: Artit Laoruengthana, MD

Department of Orthopaedics, Faculty of Medicine, Naresuan University,

99 Moo 9 Thapho, Phitsanulok 65000, Thailand

Tel: +66-851102799, Fax: +66-55965005

E-mail: artitlao@gmail.com rate ranging from $2.7 \%$ to $18 \% .{ }^{5}$ The estimated incidence of PJI after TKA is between $1 \%$ and $2 \%{ }^{5,6)}$ Although the incidence itself is low, the number of patients with this complication seems to be increasing exponentially owing to the dramatic growth of the volume of TKAs. ${ }^{7)}$

Proper preoperative skin preparation and draping, suitable antibiotic prophylaxis, shortened operative time, and sterility of surgical equipment are essential to decrease the incidence of PJI. ${ }^{5,8,9)}$ Despite implementation of these preventive strategies, PJIs have been reported, and the source of bacteria that cause PJI has not been clearly identified. Many studies have suggested contamination of the so-called sterile surgical equipment as a possible source: ${ }^{10-14)} 10 \%$ of suture needles, $14.5 \%$ of light handles, $17 \%$ of surgical gowns, and $28.7 \%$ of surgical gloves were found contaminated during joint arthroplasty proce- 
Rattanaprichavej et al. Burnt Necrotic Tissue in Total Knee Arthroplasty

Clinics in Orthopedic Surgery • Vol. 12, No. 1, $2020 \bullet$ www.ecios.org

dures. ${ }^{10)}$ In particular, suction tips are the most frequently contaminated surgical instrument with the contamination rate ranging from $13.33 \%$ to $80 \%{ }^{12,15,16)}$ Hence, it has been recommended that some surgical equipment such as gloves and suction tips should be appropriately replaced during surgery. ${ }^{17)}$

The electrocautery device has become a fundamental surgical instrument commonly used for dissecting subcutaneous tissue, muscle, and fascia and controlling bleeding in surgery. The reported bacterial contamination rate of electrocautery device ranges from $5.6 \%$ to $14.7 \% .{ }^{18-20)}$ By contrast, contamination of burnt necrotic tissue (BNT), collateral residual debris produced in the electrocoagulation process or diathermy, is not known. We hypothesized that the BNT may possibly be a reservoir of bacteria and consequently cause PJI if left in the operative field. In this study, we aimed to investigate (1) the contamination rate of BNT in the surgical field of TKA, (2) association between contamination of BNT and the incidence of PJI after TKA, and (3) different characteristics between patients with contaminated and non-contaminated BNT and between patients with PJI and without PJI.

\section{METHODS}

We conducted a retrospective cohort analysis of data from 225 consecutive patients who underwent unilateral primary TKA at our institution. Five patients with a history of prior knee infection, one patient with recent bacteremia (within 1 year), and 22 patients with previous knee surgery were excluded. Fourteen patients were additionally excluded owing to inadequate data, considering the purpose of the current study. Consequently, 183 patents (35 men and 148 women) with primary TKA were enrolled in this

\section{Table 1. Demographic Data of All Included Patients}

\begin{tabular}{lr}
\multicolumn{1}{c}{ Variable } & Outcome \\
\hline Age $(\mathrm{yr})$ & $63.9 \pm 8.6$ \\
\hline Sex (male : female) & $35: 148$ \\
\hline Body mass index $\left(\mathrm{kg} / \mathrm{m}^{2}\right)$ & $27.2 \pm 4.0$ \\
\hline Side (right : left) & $88: 95$ \\
\hline ASA physical status classification (I : II : III) & $44: 132: 7$ \\
\hline Operative time (min) & $80.2 \pm 19.1$ \\
\hline Collecting time (min) & $30.5 \pm 19.0$ \\
\hline
\end{tabular}

Values are presented as mean \pm standard deviation.

ASA: American Society of Anesthesiologists. study (Table 1). The study was approved by the Institutional Review Board of Naresuan University Hospital (IRB No. 595/60), and written informed consent was obtained from all patients.

All surgical procedures were performed with a similar surgical technique through a standard medial parapatellar approach with conventional instruments in a laminar air flow operating room. Prophylactic antibiotics were administered to every patient: $1 \mathrm{~g}$ of intravenous cefazolin at 30 minutes prior to the operation, and then $1 \mathrm{~g}$ of the same antibiotic every 8 hours for 48 hours (clindamycin was considered if patient had a history of adverse reaction to penicillin). The skin was prepared by using a combination of betadine solution and alcohol-based antiseptic according to the International Consensus Meeting (ICM) 2013. ${ }^{21,22)}$ All patients underwent spinal anesthesia with single-shot femoral nerve block. A tourniquet was applied at 300 $\mathrm{mmHg}$ to achieve a bloodless surgical field. Monopolar electrocautery (Excell NHP 400/D, Alsa Medica, Bologna, Italy) was used for soft-tissue dissection and coagulation. An identical postoperative protocol including patientcontrolled analgesia with morphine, drain and catheter management, and rehabilitation was used for all patients.

Depending on the surgeon's preference, multiple BNT specimens (3-5) were randomly collected during the operation in the surgical field. After collection, these specimens were placed in a sterile container and immediately sent to the microbiology department. Each specimen was cultured by inoculation at $35^{\circ} \mathrm{C}$ onto blood agar plate (Biomedia, Nonthaburi, Thailand), MacConkey agar plate (Biomedia), and Thioglycollate broth (Oxoid, Hampshire, UK) for 24 to 72 hours, and then visually observed for presence or absence of growth. Ten sterile electrocautery tips were cultured as a control group.

The outcome parameters consisting of demographic data, American Society of Anesthesiologists (ASA) physical status classification, operative time, and collecting time were routinely evaluated. Collecting time was defined as the duration from the start of using the electrocautery device to the first collection of a BNT specimen. All patients were followed up for a minimum of 2 postoperative years according to ICM 2013.

\section{Statistical Analysis}

Descriptive statistics such as mean and standard deviation were used as appropriate to report the outcomes. The unpaired $t$-test was used to compare the difference in characteristics between the contaminated (culture-positive) BNT group and the non-contaminated (culture-negative) BNT group and also between PJI group and non-PJI group, 
Rattanaprichavej et al. Burnt Necrotic Tissue in Total Knee Arthroplasty

Clinics in Orthopedic Surgery • Vol. 12, No. 1, $2020 \bullet$ www.ecios.org

with statistical significance defined as $p<0.05$. The SPSS ver. 17.0 (SPSS Inc., Chicago, IL, USA) was used for all analysis. Based on the $10 \%$ estimated contamination rate of electrocautery tips in patients with primary joint arthroplasty ${ }^{20)}$ and the $4.3 \%$ minimum rate of electrocautery contamination in TKA patients, ${ }^{20)}$ a post-hoc power analysis revealed that the sample size of this study has $81.7 \%$ power to detect a difference between PJI and non-PJI group with an alpha of 0.05 .

\section{RESULTS}

BNT culture was positive (contamination) in $4.4 \%$ (eight of 183 patients). The most common organism in culturepositive specimens was coagulase-negative Staphylococcus (five of eight patients, 62.5\%). Pseudomonas species (spp.), Corynebacterium spp., and Acinetobacter baumannii were the solely isolated organisms in the remaining three positive cultures (12.5\%). None of the sterile electrocautery tips was culture positive. While none of the patients with contaminated BNT showed signs of PJI at the last followup, four of the patients with non-contaminated BNT (4/179 patients, 2.2\%) developed PJI during the 2 postoperative years. The causative organism was Staphylococcus aureus in one patient and Streptococcus epidermidis in another patient, but we could not identify any organisms from fluid and tissue of the other two patients. The PJI was diagnosed early in all patients and successfully treated with debridement and a prosthesis-retaining protocol.

On comparison of the contaminated and non-contaminated BNT groups, there were no statistically significant differences in age, body mass index, and ASA classification. The contaminated BNT group had longer operative and collecting time than the non-contaminated BNT group, but the difference was not statistically significant (Table 2). There was also no difference in age, body mass index, ASA classification, and operative time between PJI and non-PJI groups (Table 3).

\section{Table 2. Comparison of Demographic and Perioperative Data between Contaminated and Non-contaminated Groups}

\begin{tabular}{lccc|}
\hline \multicolumn{1}{c}{ Variable } & Contaminated & Non-contaminated & $p$-value \\
\hline Age (yr) & $65.1 \pm 10.0$ & $63.8 \pm 8.6$ & 0.68 \\
\hline Sex (male : female) & $2: 6$ & $33: 142$ & NA \\
\hline Body mass index $\left(\mathrm{kg} / \mathrm{m}^{2}\right)$ & $27.3 \pm 2.9$ & $27.2 \pm 4.1$ & 0.94 \\
\hline Side (right : left) & $4: 4$ & $84: 91$ & $\mathrm{NA}$ \\
\hline ASA physical status classification $(\mathrm{I}: \mathrm{II}:$ III) & $3: 5: 0$ & $41: 127: 7$ & 0.31 \\
\hline Operative time (min) & $103.1 \pm 44.2$ & $79.0 \pm 16.7$ & 0.17 \\
\hline Collecting time (min) & $48.0 \pm 44.3$ & $29.7 \pm 17.0$ & 0.28 \\
\hline
\end{tabular}

Values are presented as mean \pm standard deviation.

NA: not available, ASA: American Society of Anesthesiologists.

\section{Table 3. Comparison of Demographic and Perioperative Data between PJI and Non-PJI Groups}

\begin{tabular}{lccc}
\multicolumn{1}{c}{ Variable } & PJI & Non-PJI & $p$-value \\
\hline Age $(\mathrm{yr})$ & $67.5 \pm 8.7$ & $63.8 \pm 8.6$ & 0.46 \\
\hline Sex (male : female) & $0: 4$ & $35: 144$ & $\mathrm{NA}$ \\
\hline Body mass index $\left(\mathrm{kg} / \mathrm{m}^{2}\right)$ & $29.8 \pm 7.4$ & $27.1 \pm 4.0$ & 0.51 \\
\hline Side (right : left) & $3: 1$ & $85: 94$ & $\mathrm{NA}$ \\
\hline ASA physical status classification $(I:|| \mid$ : III) & $1: 3: 0$ & $43: 129: 7$ & 0.86 \\
\hline Operative time (min) & $73.8 \pm 8.4$ & $80.2 \pm 19.3$ & 0.23 \\
\hline
\end{tabular}

Values are presented as mean \pm standard deviation.

PJl: periprosthetic joint infection, NA: not available, ASA: American Society of Anesthesiologists. 
Rattanaprichavej et al. Burnt Necrotic Tissue in Total Knee Arthroplasty

Clinics in Orthopedic Surgery • Vol. 12, No. 1, $2020 \bullet$ www.ecios.org

\section{DISCUSSION}

Sterility of surgical equipment is essential to prevent postoperative infection in contemporary orthopedic practice. Nevertheless, contamination of such regularly used "sterile equipment" has been reported in several studies. ${ }^{10-13,23)}$ In the current study, we found that $4.4 \%$ of BNT from the surgical field can be contaminated, most commonly by coagulase-negative Staphylococcus (62.5\%). The BNT, a residual product of the electrocoagulation process, is a nonviable tissue that lacks protective mechanism, and thus can be an ideal reservoir for microorganisms. Indeed, an infection might occur when the host immune response is affected by these contaminating microorganisms. The association between contamination of sterilized equipment and deep surgical site infection has been demonstrated in orthopedic and ophthalmic operations. ${ }^{23)}$ In addition, use of electrocoagulation could cause an increase in proinflammatory cytokines, which was well recognized at 48 hours after surgery in previous studies, ${ }^{24,25}$ and the elevation of inflammation level may consequently lead to another infection. Our findings show that it is impossible to ensure an absolute microorganism-free environment/ equipment during primary TKA; however, the contaminated BNT from the clean primary TKA field was not associated with the surgical site infection.

Although there has been no previous study on the contamination of BNT, it might be related to electrocoagulation. Shahi et al. ${ }^{18)}$ assessed a series of 50 cultures from electrocautery tips after total hip arthroplasty. They reported a $6 \%$ positive culture rate, but none of the patients developed PJI. The estimated blood loss and electrocautery tip exposure time did not show a significant difference between the contaminated and non-contaminated groups in their study. Abdulkarim et al. ${ }^{19)}$ also reported a 5.6\% contamination rate of the diathermy tips after orthopedic surgery. Recently, Abdelaziz et al. ${ }^{20)}$ demonstrated a $10 \%$ contamination rate of electrocautery tips in primary total hip and knee arthroplasty, and the incidence was up to $30 \%$ in septic revision. As in our study, Staphylococcus epidermidis, a member of coagulase-negative Staphylococcus groups, was the most commonly isolated organism in their study. Furthermore, $80 \%$ of contaminated electrocautery tips had the same causative microorganism in single-stage exchange caused by PJI. They also found an insignificant relationship between the duration of use or exposure time and the contamination rate of the electrocautery tip.

Heat is generally used to decontaminate or sterilize surgical equipment to minimize the risk of infection caused by microorganisms. However, regardless of the sterilization technique, temperature and duration are regarded as the most important factors in the sterilization process. The standard steam sterilization technique guided by the Center of Disease Control requires 30 minutes of exposure at $121^{\circ} \mathrm{C}$ in a gravity displacement sterilizer or 4 minutes of exposure at $132^{\circ} \mathrm{C}$ in a prevacuum sterilizer. ${ }^{24)}$ Although the cautery tip can be as hot as $200^{\circ} \mathrm{C}$ in some settings, the continuous heating/exposure time is scarcely less than 30 minutes and the heat from cautery tips may be reduced while being transferred to BNT. Therefore, subsequent sterility of the electrocautery device with selfproducing heat may not be guaranteed. ${ }^{25,26)}$

There were some limitations of our study. First, this study is a retrospective cohort study with the limitation inherent to the study design. However, our 2-year followup evaluation might provide useful clinical relevance by showing no association between bacterial contamination of BNT and the development of PJI. Second, our findings may only be pertinent to the primary TKA setting. In revision surgery, the contamination rate of electrocautery tips is higher, particularly in septic revision. ${ }^{20)}$ Hence, residual product, or BNT, may also be at greater risk of contamination. Additionally, extensive electrocoagulation that aims to control bleeding surface during TKA or other procedures such as spinal surgery may increase the amount of BNT and level of inflammation. ${ }^{27,28)}$ Then, an investigation on the contamination of BNT and its association with PJI in these circumstances may yield different results, and thus further research might be warranted. Last, BNT in the present study might have been contaminated by other surgical instruments because normal tissue and electrocautery tips from the surgical field had not been taken for culture. In conclusion, BNT in the surgical field can become a reservoir of contaminating bacteria in primary TKA. However, the contamination of BNT was not directly associated with PJI. Therefore, routine removal of all BNTs or change to a new electrocautery tip may be unnecessary in primary TKA.

\section{CONFLICT OF INTEREST}

No potential conflict of interest relevant to this article was reported.

\section{ACKNOWLEDGEMENTS}

We thank Kwankaew Wongchareon, PhD (Faculty of Nursing, Naresuan University, Phitsanulok, Thailand) and Krit Pongpirul, PhD (Faculty of Medicine, Chulalongkorn University, Bangkok, Thailand) for data analysis, 
Rattanaprichavej et al. Burnt Necrotic Tissue in Total Knee Arthroplasty

Clinics in Orthopedic Surgery • Vol. 12, No. 1, $2020 \bullet$ www.ecios.org

Pariphat Chompoonutprapa, MD (Faculty of Medicine, Naresuan University, Phitsanulok, Thailand), Thanawat Tantimethanon, MD (Faculty of Medicine, Naresuan University, Phitsanulok, Thailand), and Watcharapong Eiam- jumras, MD (Faculty of Medicine, Naresuan University, Phitsanulok,Thailand) for data collection, and Richard M. Glover (Faculty of Humanity, Naresuan University, Phitsanulok, Thailand) for proofreading.

\section{REFERENCES}

1. Carr AJ, Robertsson O, Graves S, et al. Knee replacement. Lancet. 2012;379(9823):1331-40.

2. Weinstein AM, Rome BN, Reichmann WM, et al. Estimating the burden of total knee replacement in the United States. J Bone Joint Surg Am. 2013;95(5):385-92.

3. Chang SY, Lin LH, Lin PC. Knee joint function, walking ability and quality of life within 6 weeks after total knee arthroplasty: a prospective cohort study. J Clin Nurs. 2019;28(17-18):3222-32.

4. Bozic KJ, Kurtz SM, Lau E, et al. The epidemiology of revision total knee arthroplasty in the United States. Clin Orthop Relat Res. 2010;468(1):45-51.

5. Matar WY, Jafari SM, Restrepo C, Austin M, Purtill JJ, Parvizi J. Preventing infection in total joint arthroplasty. J Bone Joint Surg Am. 2010;92 Suppl 2:36-46.

6. Kurtz SM, Ong KL, Lau E, Bozic KJ, Berry D, Parvizi J. Prosthetic joint infection risk after TKA in the Medicare population. Clin Orthop Relat Res. 2010;468(1):52-6.

7. Kurtz S, Ong K, Lau E, Mowat F, Halpern M. Projections of primary and revision hip and knee arthroplasty in the United States from 2005 to 2030. J Bone Joint Surg Am. 2007;89(4):780-5.

8. Kapadia BH, Berg RA, Daley JA, Fritz J, Bhave A, Mont MA. Periprosthetic joint infection. Lancet. 2016;387(10016):38694.

9. de Lissovoy G, Fraeman K, Hutchins V, Murphy D, Song D, Vaughn BB. Surgical site infection: incidence and impact on hospital utilization and treatment costs. Am J Infect Control. 2009;37(5):387-97.

10. Davis N, Curry A, Gambhir AK, et al. Intraoperative bacterial contamination in operations for joint replacement. J Bone Joint Surg Br. 1999;81(5):886-9.

11. Pinto FM, de Souza RQ, da Silva CB, Mimica LM, Graziano KU. Analysis of the microbial load in instruments used in orthopedic surgeries. Am J Infect Control. 2010;38(3):22933.

12. Al Laham NA. Prevalence of bacterial contamination in general operating theaters in selected hospitals in the Gaza Strip, Palestine. J Infect Public Health. 2012;5(1):43-51.

13. Cloutman-Green E, Canales M, Zhou Q, Ciric L, Hartley JC,
McDonnell G. Biochemical and microbial contamination of surgical devices: a quantitative analysis. Am J Infect Control. 2015;43(6):659-61.

14. Beldame J, Lagrave B, Lievain L, Lefebvre B, Frebourg N, Dujardin F. Surgical glove bacterial contamination and perforation during total hip arthroplasty implantation: when gloves should be changed. Orthop Traumatol Surg Res. 2012;98(4):432-40.

15. Givissis P, Karataglis D, Antonarakos P, Symeonidis PD, Christodoulou A. Suction during orthopaedic surgery: how safe is the suction tip? Acta Orthop Belg. 2008;74(4):531-3.

16. Larsson J, Sutherland S, Soderstrom A, et al. Bacterial contamination of suction catheter tips during aortic valve replacement surgery: a prospective observational cohort study. Patient Saf Surg. 2015;9:17.

17. Parvizi J, Gehrke T, Chen AF. Proceedings of the international consensus on periprosthetic joint infection. Bone Joint J. 2013;95(11):1450-2.

18. Shahi A, Chen AF, McKenna PB, et al. Bacterial contamination in tips of electrocautery devices during total hip arthroplasty. J Arthroplasty. 2015;30(8):1410-3.

19. Abdulkarim A, Moriarity A, Coffey P, Sheehan E. Bacterial contamination of diathermy tips used during orthopaedic procedures. Orthop Proc. 2016;98 (SUPP 1):1.

20. Abdelaziz H, Zahar A, Lausmann C, et al. High bacterial contamination rate of electrocautery tips during total hip and knee arthroplasty. Int Orthop. 2018;42(4):755-60.

21. Hansen E, Belden K, Silibovsky R, et al. Perioperative antibiotics. J Arthroplasty. 2014;29(2 Suppl):29-48.

22. Tokarski AT, Blaha D, Mont MA, et al. Perioperative skin preparation. J Arthroplasty. 2014;29(2 Suppl):26-8.

23. Dancer SJ, Stewart M, Coulombe C, Gregori A, Virdi M. Surgical site infections linked to contaminated surgical instruments. J Hosp Infect. 2012;81(4):231-8.

24. Rutala WA, Weber DJ. CDC: guideline for disinfection and sterilization in healthcare facilities (2008). Atlanta, GA: Centers for Disease Control and Prevention; 2017 [cited 2019 Dec 20]. Available from: https://www.cdc.gov/infectioncontrol/guidelines/disinfection/.

25. Barada K, Mourad FH, Noutsi B, Saade NE. Electrocautery- 
Rattanaprichavej et al. Burnt Necrotic Tissue in Total Knee Arthroplasty

Clinics in Orthopedic Surgery • Vol. 12, No. 1, $2020 \bullet$ www.ecios.org

induced localized colonic injury elicits increased levels of pro-inflammatory cytokines in small bowel and decreases jejunal alanine absorption. Cytokine. 2015;71(1):109-18.

26. Sebben JE. The hazards of electrosurgery. J Am Acad Dermatol. 1987;16(4):869-72.

27. Tammachote N, Kanitnate S. Electric cautery does not reduce blood loss in primary total knee arthroplasty com- pared with scalpel only surgery a double-blinded randomized controlled trial. Int Orthop. 2018;42(12):2755-60.

28. Seviciu A, Gross I, Fathima S, Walsh SM. Effects of tranexamic acid and bipolar sealer alone or in combination in primary total knee arthroplasty: a prospective, randomized, controlled trial. Arthroplast Today. 2016;2(2):77-82. 